280 MeGow in: Ueb. d. Abkomml. d. Methylsulfonsäure,

\title{
Ueber die Abkömmlinge der Methylsulfonsäure, insbesondere des Trichlormethylsulfonchlorids;
}

von

\section{G. Mi Gowan.}

Im März dieses Jahres ist von mir in diesem Journal eine kurze vorläufige Notiz veröffentlicht, betitelt: „Zur Kenntniss des Trichlormethylsulfonchlorids", dessen nähere Untersuchung ich, auf Veranlassung des Herrn Professor Kolbe, unternommen habe. ${ }^{1}$ ) Seitdem ist von mir die Untersuchung fortgeführt worden.

Wie schon in jener Notiz erwähnt, ist seit den von $\mathrm{Kolbe}^{2}$ ) vor ungefähr vierzig Jahren ausgeführten Untersuchungen über diese interessante Verbindung, zwei bemerkenswerthe Mittheilungen von $\mathrm{O} . \mathrm{Loew}^{3}$ ) und einige Notizen von Carius $^{4}$ ) ausgenommen, nichts veröffentlicht worden.

Vom Trichlormethylsulfonchlorid haben meine Studien sich auf Methylsulfonchlorid und Dichlormethylsulfonchlorid: resp. auf die entsprechenden Säuren, erstreckt.

Im Folgenden werde ich zunächst das Verhalten dieser Verbindungen gegen verschiedene Agentien beschreiben und einer vergleichenden Untersuchung unterwerfen.

\section{Methylsulfonsäure: $\mathrm{CH}_{3} \mathrm{SO}_{2} \mathrm{OH}$.}

Diese Säure, der Ausgangspunkt für die Darstellung des Methylsulfonchlorids, wurde durch Oxydation von Schwefelcyanmethyl mittelst Salpetersäure von 1,25 spec. Gew. gewonnen. Der Ueberschuss von Salpetersäure wurde durch wiederholten Zusatz von Wasser und Abdampfen auf dem Wasserbade verjagt. Aus dem rückständigen Produkte wurde

1) Herr Prof. Kolbe hatte die Freundlichkeit, mir eine grosst: Menge des Chlorids zur Verfügung zu stellen.

2) Ann. Chem. Pharm. 54, 145.

3) Zeitschr. Chem. 1868, S. 518; 1869, S. 82.

4) Ann. Chem. Pharm. 111, 93; 114, 140. 
insbesondere des Trichlormethylsulfonchlorids. 281

$$
\text { Methylsulfonchlorid: } \mathrm{CH}_{3} \mathrm{SO}_{2} \mathrm{Cl}
$$

durch Einwirkung von Fünffach-Chlorphosphor und nachheriges Fractioniren gewonnell.3) Das spec. Gew. dieses Chlorids beträgt annähernd 1,51 .

Einwirkung von Ammoniak auf Methylsulfonchlorid.

Trocknes Ammoniakgas, in eincm graduirten Rohre über Quecksilber aufgefangen, wurde von einer aetherischen Lösung des Chorids vollständig absorbirt unter Bildung des Methylsulfonamids. Durch Einwirkung von wässrigem unl absolutem alkoholischen Ammoniak auf das Chlorid wurdo kein Stickstoff frei (vergl. das Verhalten des Trichlormethylsulfonchlorids).

\section{Methylsulfonamid: $\mathrm{CH}_{3} \mathrm{SO}_{2} \mathrm{NH}_{2}$.}

Diese Verbindung wurde erhalten durch Sättigung einer nicht zu concentrirten Lösung des Chlorids in absolutem Aether mit trocknem Ammoniak, wobei sich ziemlich viel Wärme entwickelt. Die resultirende feste Masse wurde zuerst mit einem Gemisch von Alkohol und Benzol ausgezogen und schliesslich aus kochendem Benzol - welches eine kleine Menge Alkohol enthielt - krystallisirt. Aus diesem schied es sich beim Erkalten in langen gestreiften und vielfach gekreuzten Prismen aus.

$$
\text { Analyse: }
$$

0,2160 Grm. gaben 27,05 Ccm. $\mathbf{N}$ bei $755 \mathrm{Mm}$. Bar. und $10^{\circ}$.

$$
\begin{array}{cc}
\text { Berechnet } & \text { Gefunden. } \\
\text { für } \mathrm{CH}_{3} \mathrm{SO}_{2} \mathrm{NH}_{2} . & 14,90 \%
\end{array}
$$

Mit Salzsäuregas vereinigt sich das Methylsulfonamid nicht.

Methylsulfonsaures Ammon: $\mathrm{CH}_{3} \mathrm{SO}_{2} \mathrm{ONH}_{4}$.

Dieses Salz krystallisirt leicht aus kochendem absoluten Alkohol beim Erkalten in dünnen rhombischen Blättern.

1) Vergl. Carius, Ann. Chem. Pharm. 114, 142. 
282 Mo Gowan: Ueb. d. Alskömml, d. Methylsulfonsäure,

In Wasser ist es leicht, in kochendem Alkohol ziemlich, in kaltem aber schr schwer löslich.

Analyse:

0,2585 Grin. gaben 27,3 Cem. $\mathrm{N}$ bei $754 \mathrm{Mm}$. Bar. und $14^{\circ}$.

$$
\begin{aligned}
& \text { Berechnet Gefuncien. } \\
& \text { fiir } \mathrm{CH}_{3} \mathrm{SO}_{2} \mathrm{ONH} \mathrm{N}_{4} \text {. } \\
& \mathrm{N}=12,39 \%
\end{aligned}
$$

Methylsulfonanilid: $\mathrm{CH}_{3} \mathrm{SO}_{3} \mathrm{NH}\left(\mathrm{C}_{6} \mathrm{H}_{5}\right)$.

Auf tropfenweissen Zusatz von Anilin zu Methylsulfonchlorid entsteht unter Ausgabe von viel Wärme eine festc Masse. Es ist rathsam, einen kleinen Ueberschuss von Anilin anzuwenden. Die Reaction wird auf dem Wasserbade beendet. Giesst man die in wenig Alkohol gelöste feste Masse in viel Wasser, so scheidet sich zunächst ein Oel aus, welches dann sogleich krystallinisch erstarrt. Dieses Produkt lässt sich leicht aus Alkohol rein erhalten. Das zu gleicher Zeit gebildete salzsaure Anilin bleibt in dem Wasser aufgelöst.

$$
\text { Analyso: }
$$

0,403 Grm. gaben 28,85 Ceno. N bei $763 \mathrm{Mm}$. Bar. und $11^{0}$.

$$
\begin{aligned}
& \text { Berechnet } \\
& \mathrm{N} \text { für } \mathrm{CH}_{3} \mathrm{sO}, \mathrm{NH}\left(\mathrm{C}_{6} \mathrm{H}_{5}\right), \\
& =8,2 \% \%
\end{aligned}
$$

Das Methylsulfonanilid ist - wie schon erwähnt - in Wasser schwer, lcicht in Alkohol löslich, aus welchem es in grossen schönen Blättern, (sehr wahrscheinlich rhombisch, möglicherweise aber monoklinisch), anskrystallisirt.')

1) Anmerkung des Herrn Prof. Zirkel über die Krystallform dieses Anilids: ,Die Form-Ausbildung und die optischen Eigenschaften der Krystalle lassen nur darüber einen Zweifel, ob sie dem rhombischen oder monoklinen system angehören."

"Auf die breiten Tafelfächen golegt, erweisen sie sich - zwischen gekreuzten Nicols - vollkommen gerade auslöschend. Bei monoklinen Krystallen müsste diese Fläche stets dem Orthopinakoid $\infty$ P $\infty$ entsprechen. Der Habitus der Krystalle unter dem Mikroskop scheint aber vielmehr auf das rhombische System zu verweisen: auf eine Combination zweier Pinakoide mit zwei Domen, oder einem Dorna und 
insbesondere des 'Trichlormethylsulfonchlorids. 283

Schwefelwasserstoff übt auf die Lösung des Methylsulfonchlorids in viel absolutem Alkohol keine reducirende Einwirkung. Der Schwefelwasserstoff wurde zuvor mit Wassergewaschen und mittelst Chlorcalcium getrocknet. Nachdem das Gias mehrere Stunden durch die Lösung hindurchgegangen war, wurde die Flasche, fest verschlossen, einige Tage stehen gelassen; es fand keine Ausscheidung von Schwefel statt.

Einwirkung von Cyankalium auf Methylsulfonchlorid.

Alle Versuche, Methylsulfoncyanid: $\mathrm{CH}_{3} \mathrm{SO}_{3} \mathrm{CN}$ durch Einwirkung von Cyankalium auf das in Alkohol gelösto Methylsulfonchlorid darzustellen, waren fruchtlos.

Ferner wurde die Einwirktng des Cyankalium auf das Chlorid in folgender Weise geprïft: in Benzollösung bei Siedetemperatur, sowie in zugeschmolzenen Röhren bei Temperaturen von $105^{\circ}$ und $180^{\circ}$, endlich ohne irgend welches Lösungsmittel in einem zugeschmolzenen Rohre bis auf $100^{\circ}$. Bei allen diesen Versuchen wurde nach Zufügen des Chlorids zu dem Cyankalium Wärme entwickelt und eine braun oder schwarz gefärbte Substanz gebildet. Ein kleiner Theil des Cyankaliums ging in Chlorkalium über.

Versuche, die Methylsulfonsäure, sowie deren Chlorid zu chloriren, sind ohne Erfolg geblieben. Dieselben mögen hier kurz erwähnt werden, da sich daraus die ausserordentliche Beständigkeit beider Körper ergiebt:

a. Die Säure wurde zuerst für sich allein und dann unter Zusatz von Jod der mehrstündigen Einwirkung von Chlor im hellen Sonnenlichte ausgesetzt.

b. Methylsulfonchlorid wurde mit Dreifach-Chlorjod in einem geschlossenen Rohre bis zu $120^{\circ}$, und weiter bis auf $200^{\circ}$ erhitzt.

c. Ein Gemisch von,Chlorid und Säure zusammen wurde mit Dreifach-Chlorjod auf $190^{\circ}$ erhitzt.

d. Das Chlorid wurde mit Sulfurylchlorid: $\mathrm{SO}_{2} \mathrm{Cl}_{2}$ auf $130^{\circ}$ und weiter bis auf $210^{\circ}$ erhitzt.

einem Prisma. Die Zugehörigkeit zu dem triklinen System iit optisch ausgeschlossen." 
284 MeGowan: Ueb. d. Ablömml. d. Methylsulfonsäure,

Man erkennt leicht, ob Chlorirung stattgefunden hat oder wicht, denn das aus dem Produkte dargestellte methylsulfonsuture Kali krystallisirt aus kochendem Alkohol beim Erkatten, als Magma sehr feiner Nadeln aus.

\section{Trichlormethylsulfonsüure: $\mathrm{CCl}_{3} \mathrm{SO}_{2} \mathrm{OH}$.}

Zur Darstellung dieser von Kolbe - unter dem Namen Chlorkohlenunterschwefolsäure _ beschriebenen Säure 1) wird zunächst das Barytsalz durch Digeriren des Trichlormethylsulfonchlorids mit eiuer concentrirten Lösung von Barythydrat bereitet. Diese Reaction gelingt am besten bei Anwendung von nur selir wenig Wasser. Zu einer Operation brauchte ich ungefähr 100-200 Grm. Chlorid auf 150-350 Grm. krystallisirtes Barythydrat. Eine erhebliche Menge schwefelsauren Baryts wird jedesmal dabei ausgeschieden. ${ }^{2}$ )

Nachdem die Einwirkung beendet ist, werden alle in der Lösung enthaltenen Barytsalze durch verdünnte Schwefelsäure gefailt, der Ueberschuss dieser Säure durch kohlensaures Blei und der Ueberschuss Blei durch Schwefelwasserstofi. Da es nicht nothwendig ist, das Kalisalz darzustellen und daraus die Säure wieder abzuscheiden, so verdampfte ich einfach das Filtrat vom Schwefelblei auf dem Wasserbade --.- unter öfterem Zusatz von Wasser - bis alle Salzsäure verjagt war. Auf diese Weise bekommt man die Trichlormethylsulfonsäure genügend reiı. Um zu prüfen ob die so dargestellte Säure rein war, neutralisirte ich eine kleine Quantität davon mit Katlilauge, verdunstete die Lösung bis zur Trockene und trocknete das so gewonnene Salz zunächst zwischen Fliesspapier und dann über Schwefelsäure im Vacuum.

Analyse:

$0,6072 \mathrm{Grm}$. gaben $0,2446 \mathrm{Grm} . \mathrm{K}_{2} \mathrm{SO}_{1}$.

1) Ann. Chem. Pharm. 54, 145.

3) Anch eine kleine Quantitit einer ätherischen Flüssigkeit bildet sich, welche schwerer als Wasser ist: nicht unwahrscheinlich ein Chlorkohlenstoff, vielleicht 'Tetrachlorkohlenstoft'. 
insbesondere des Trichlormethylsulfonchlorids. 285

\author{
Berechnet \\ für $\mathrm{CCl}_{3} \mathrm{SO}_{2} \mathrm{OK}$. \\ $\mathrm{K}=16,43 \% \quad \mathbf{1 6 , 6 5 \%}$
}

Ueber die Einwirkung einiger Metalle auf Trichlormethylsulfonsäure.

1) Die Einwirkung von Zink auf diese Säure ist schon von $\mathrm{Kolbe}^{\mathrm{l}}$ ) ausführlich beschrieben worden; ich werle weiter unten auf dieselbe zurückkommen.

2) Silberschwamm wird weder von einer kalten, noch von einer heissen wässerigen Lösung der Säure angegriffen.

3) Eisenfeile löst sich in der wässerigen Lösung ler Säure unter Entwickelung von Wasserstoff $\mathrm{zu}$ einer blassgrünen Flüssigkeit auf. Wird, nachdem die Einwirkung beendet ist, die abfiltrirte Lösung eingedampft, anfangs bei gelinder Wärme auf dem Wasserbade ${ }^{2}$ ) und nachher im Vacuum über Schwefelsäure (siehe unten), so krystallisirt ein Salz in sehr schönen langen durchsichtigen hellgrünen Prismen, welche gestreift, aber nicht zugespitzt sind.

Analyse des Eisensalzes:

Eine Analyse dieser zwischen Fliesspapier (nicht über Schwef(tsüure in Vacuum) getrockneten Krystalle gab folgende Resultate:

a. Wasserverlust bei $100^{\circ}$ :

$0,3121 \mathrm{Grm}$. verloren bei $100^{\circ} 0,0213 \mathrm{Grm}$. Wasser $=6,82 \%$ vom Gewicht des Salzes.

Berechnet für $\left(\mathrm{CCl}_{3} \mathrm{SO}_{2} \mathrm{O}\right)_{2} \mathrm{Fe}+5 \mathrm{H}_{2} \mathrm{O}$, welches in $\left(\mathrm{CCl}_{3} \mathrm{SO}_{2} \mathrm{O}\right)_{2} \mathrm{Fe}$ $+3 \mathrm{H}_{2} \mathrm{O}$ übergergangen ist: $6,63 \%$.

b. Wasserverlust bei $\left.185^{\circ}-190^{\circ 3}\right)$ :

Diese Bestimmung wurde in einem $U$-förmigen Rohre, wolches in einem Schwefelsüurebad unter Durchleiten von einem Strom Kohlensäure erhitzt wurde, gemacht.

0,3891 Grm. verloren beim Erhitzen während etwa vier Stunien bis auf $185^{\circ}-190^{\circ} 0,0715$ Grm. Wasser.

1) Ann. Chem. Pharm. 54, 145.

2) Während dicses Eindampfeus scheidet sich etwas rothbraune Substanz aus der Fliissigkeit ab; diese wird wahrscheinlich ein basisches Eisenoxydsalz sein.

3) Eine niedrigere Temperatur würde vielleicht genügen. 
286 I`G o wan: Ueb. d. Abkömml. d. Methylsulfonsäure,

$\begin{array}{lc}\text { Rerechnet } & \text { Gefunden. } \\ \mathrm{H}_{2} \mathrm{O} & 18,37 \% \\ \text { c. Eisenbestimmung: } & 18,48 \%\end{array}$

0,535 Grm. in Wasser, welchem ein wenigr salzsäure angefügt war, aufgelöst, duneh Salpetersäure oxydirt, und dann heiss durch Ammoniak niedergesehiagen, gaben 0,0748 Grm. $\mathrm{Fe}_{2} \mathrm{O}_{3}$.

$$
\begin{aligned}
& \text { Berechmet Gefunden. } \\
& \text { für }\left(\mathrm{COH}_{3} \mathrm{SO}_{2} \mathrm{O}\right)_{2} \mathrm{Fc}+5 \mathrm{H}_{2} \mathrm{O} \text {. } \\
& \text { Fe } \quad 10,31 \% \quad 10,40 \%
\end{aligned}
$$

d. Dieses Salz verliert auch zwei Moleküle stines Krystallwassers nicht nur bei erhöhter 'Temperatur, sondern auch, wenn es eine kurze Zeit über Schwefelsture in Vacuum aufbewahrt wird. Die durehsichtigen eisengrünen Krystalle werden dadureh ondurcbsichtig und von blass citronengelber Farbe.

0,4878 Grm. des Salzes, so getrocknet, gaben 0,0760 Grm. Fe $\mathrm{O}_{2}$. Berechnet Gefunden. für $\left(\mathrm{CCl}_{3} \mathrm{SO}_{2} \mathrm{O}\right)_{2} \mathrm{Fe}+3 \mathrm{H}_{2} \mathrm{O}$.

Fe $\quad 11,05 \%_{0} \quad 10,91 \%$

Trichlormethylsulfonsaures Eisen, einmal getrocknet, scheint verhältnissmässig beständig zu sein; in Lösung erfährt es natürlicherweise leicht Oxydation.

4) Feines Zinnblech wird von einer kalten wässerigen İösung der Trichlormethylsulfonsäure scheinbar nicht angegriffen. Bei dem Erwärmen jedoch auf dem Wasserbade löst sich das Zinn ${ }^{1}$ ) allmählich auf und zwar in dem Verhältnisse von einem Atom Zinn zu einem Molecül Säure. ${ }^{2}$ )

Daraus folgt, dass auch hier, wie bei der Anwendung von Zink eine reducirende Einwirkung auf die Trichlormethylsulfonsäure stattgefunden hat, unter Bildung des Zinnsalzes der Trichlornethylsulfonsäure (vergl. die Einwirkung des Zinks weiter unten).

Trichlormethylsulfonchlorid: $\mathrm{CCl}_{3} \mathrm{SO}_{2} \mathrm{Cl}$.

Carius $^{3}$, hatte schon versucht, dieses Chlorid nach der allgemeinen Methode zur Darstellung von Säurechloriden,

1) Unter Wasserstoffentwicklung.

2) 3,52 Grm. Säure lösten 1,93 Grm. Zinn auf. Die berechnete Menge ist 2,07 Grm.

3) A. a. 0. 
insbesondere des Trichlormethylsulfonchlorids. 287

nämlich durch die Einwirkung von Fünffach-Chlorphosphor auf Trichlormethylsulfonsäure zu gewinnen, jedoch ohne Erfolg. Auch meine Versuche in dieser Richtung sind umsonst gewesen.

Fünffach-Chlorphosphor wirkt auf Trichlormethylsulfo:säure bei gewöhnlicher Temperatur ein, und diese Finwirkung kann durch Erwärmen auf dem Wasserbade beschleunigt werden. Das Produkt der Reaction ist, nach Trennung des Phosphoroxychlorids, eine schwere aetherische, zugleich stechend riechende Flüssigkeit ${ }^{1}$ ); keine Spur Trichlormethylsulfonchlorid war nachzuweisen. - Wird die Süure mit Finnfach-Chlorphosphor in einem zugeschmolzenen Rohre bis auf $130^{\circ}$ erhitzt, so spaltet sich ihr Molecül in schweflige Säure und wahrscheinlich einen Chlorkohlenstoff $\left(\mathrm{CCl}_{4}\right.$ ?). Bei einem anderen Versuch schien Carbonylchlorid: $\mathrm{COCl}_{2}$ gebildet zu sein.

Das Kalisalz: $\mathrm{CCl}_{3} \mathrm{SO}_{2} \mathrm{OK}$ wird von Fünffach-Chlorphosphor beim Erhitzen bis auf $160^{\circ}$ nicht angegriffen, wenn nicht Jod dabei ist, in welch letzteren Falle es immer bei niederer Temperatur zersetzt wird; bei $250^{\circ}$ jedoch erfäht os Zerlegung in derselben Weise wie oben für die Säure angegeben worden ist.

Das von mir angewandte Trichlormethylsulfonchlorid war durch die im Tageslicht länger andauernde Einwirkung von nascirendem Chlor (einem Gemisch von Mangansuperoxyd und Salzsäure) und Salpetersäure auf Schwefelkohlenstoff dargestellt. ${ }^{2}$ ) Wenn das Chlorid rein gewünscht wird, so muss es aus Benzollösung, welche zuvor mit Chlorcaleium getrocknet war, krystallisirt werden, (oder diese filtrirte Lösung zur Trockne eingedampft), nicht aus Alkohol oder Aether, da es auf diese beiden, selbst bei gewöhnlicher Temperatur, eine geringe Einwirkung ausübt, wodurch das so gewonnene Chlorid allemal mit mehr oder weniger Salzsäure verunreinigt wird. Dieses Chlorid löst sich in kochender.

1) Diese könnte noch eine kleine Menge Phasphoroxychlorid enthalten haben. (?)

9) Siche Kolbc, Ann. Chem. Pharm. 54, 145. 
288 Me Gowan: Ueb. d. Abkőmml. d. Methylsulfonsäure,

rauchender Salpetersäure auf; nach Zusatz von Wasser zu dieser Lösung fällt es unverändert wieder aus.

Einwirkung von Cyankalium auf Trichlormethylsulfonchlorid in alkobolischer Lösung.

Loew') hat die Einwirkung von Cyankalium in wässriger: Lösung auf dieses Chlorid untersucht und drückt sie durch folgende zwei Gleichungen, welche gleichzeitig stattfinden, aus.

1) $\mathrm{CCl}_{3} \mathrm{SO}_{2} \mathrm{Cl}+\mathrm{KCN}=\mathrm{CNOl}+\mathrm{CCl}_{3} \mathrm{SOOK}$.

Trichlormethylsulfinsaures Kali.

2) $\left.\mathrm{CCl}_{3} \mathrm{SOOK}+\mathrm{H}_{9}()^{2}\right)=\mathrm{HCl}+\mathrm{CCl}_{2} \mathrm{OH} \mathrm{SOOK}$.

Dichloroxymethylsulfinsaures Kali.

In einer absolut alkoholischen Lösung ist der Gäng der Reaction derselbe, mit dem Unterschied, dass, wenn die Lösung nicht zu concentrirt ist, kein Paracyan gebildet wird, (wie bei Loew's Versuchen der Fall war).

Wenn zu einem Ueberschuss von fein zerriebenem Cyankalium in einer genügenden Menge absoluten Alkohols das Chlorid allmählich hinzugefügt wird, so erwärmt sich die Flüssigkeit, wird gelb bis bräunlich, und Chlorcyan entwickelt sich. $^{3}$ )

Die Reaction wurde auf dem Wasserbade beendet. Es ist schwer, das Dichloroxysulfinsallz durch Auskrystallisiren uus absolutem Alkohol vollständig rein zu erhalten.

1) Zeitschr. Chem. 1868, s. 518 .

2) Z. B. alkalisches Wasser.

3) Das Freiwerden des Chlorcyans wurde folgendermaassen bewiesen: das sich entwickelnde Gas, durch eine alkoholische Kalilösung hindurehgeleitet, sehied Chlorkalium aus. - Die abfiltrirte Flüssigkeit wurde dann beinahe bis zur Trockne in ciner silbernen Schale verdunstet, der Rückstand mit etwas Wasser aufgenommen, und verdünnte Schwefelsäure hinzugefügt. Zusatz von Natronlauge verursachte nun eino Entwicklung von Ammoniak, welehes von Cyansäure herrïhren musste. 
inshesondere des Trichlormethylsulfonchlorids. 289

Analyse:

a. 0,2687 Grm., zuerst für sich gelinde erhitzt und dann mit Schwefelsäure in einem Platintiegel geglüht, gaben 0,1235 Grm. $\mathrm{K}_{2} \mathrm{SO}_{4}$.

Berechnet Gefunden. für $\mathrm{CCl}_{2} \mathrm{OHSOOK}$.

K $\quad 19,21 \% \quad 19,32 \%$

b. 0,4035 Grm., mit reinem Kalk geglüht, gaben 0,5776 Grm. AgCl $=0,1429 \mathrm{Grm} . \mathrm{Cl}$.

Berechnet Gefunden.

für $\mathrm{CCl}_{2} \mathrm{OHSOOK}$.

Cl $\quad 34,98 \% \quad 35,41 \%$

Ausser obigem Dichloroxymethylsulfinsalz wird auch hier, wie schon L o ew beobachtete, zu gleicher Zeit eine grosse Quantität des trichlormethylsulfinsauren Kalis $\mathrm{CCl}_{3} \mathrm{SOOK}$ gebildet. Die Ausbeute an ersterem Salze ist nie sehr gross; es scheint darauf anzukommen, dass die Lösung nicht zu concentrirt sei, und dass die Einwirkung nicht zu rasch fortschreite.

Dichloroxymethylsulfonchlorid: $\mathrm{CCl}_{2} \mathrm{OHSO}_{2} \mathrm{Cl}$.

Fünffach-Chlorphosphor wirkt auf obiges Salz unter Bildung des entsprechenden Dichlormethylsulfonchlorids ein. Die Reaction wird durch gelindes Erwärmen vollendet. Da die Substanz zum Fractioniren nicht hinreichte, so musste las Phosphoroxychlorid durch Schütteln mit Eis entfernt werden. Auf diese Weise wurde eine kleine Quantität einer schweren Flüssigkeit - das Chlorid - erhalten, welche ich mittelst Chlorcalcium trocknete. Es hatte einen eigenthïmlichen nicht sehr stechenden Geruch. Da ich nur eine kleine Menge davon besass, so versuchte ich nicht, dasselbe vollstïndig zu reinigen, sondern führte es sogleich in das entsprechende Anilid über.

Dichloroxymethylsulfonanilid: $\mathrm{CCl}_{2} \mathrm{OHSO}_{2} \mathrm{NH}\left(\mathrm{C}_{6} \mathrm{H}_{5}\right)$.

Dieses wurde dargestellt durch Hinzufügen eines kleinen Ueberschusses von Anilid zum obigen Chlorid; die Einwirkung erfolgt sogleich unter Wärmeentwicklung. Das Anilid konnte von dem zugleich gebildeten salzsauren Anilin leicht getrennt werden, da es in Wasser wenig löslich ist; von Benzol wird Journal f, prakt. Chemie [2] Bd. $\mathbf{3 0}$. 
290 MoGowan: Ueb. d. Abkomml. d. Methylsulfonsäure, es auch sehr wenig, von Alkohol leicht gelöst, aus welchem. letzteren es sich in sehr schönen grossen rhomboëdrischen Krystallen ansscheidet, deren Ecken abwechselnd abgestumpft sind.

Analyse:

0,2945 Grm. gaben 13,8 Ccm. N bei $751 \mathrm{Mm}$. Bar. und $10^{\circ}$.

\begin{tabular}{|c|c|}
\hline $\begin{array}{c}\text { Berechnet } \\
\text { für } \mathrm{CCl}_{2} \mathrm{OHSO}_{2} \mathrm{NH}\left(\mathrm{C}_{6} \mathrm{H}_{5}\right) .\end{array}$ & Gefunden. \\
\hline $\mathrm{N} \quad 5,47 \%$ & $5,54 \%$ \\
\hline
\end{tabular}

Einwirkung von Schwefelwasserstoff und von schwefliger Säure auf Trichlormethylsulfonchlorid.

Diese Reaction ist schon eingehend von $\mathrm{K}_{0} / \mathrm{be}$ und von Loew untersucht worden; mit dem Resultat, dass das Trichlormethylsulfonchlorid in alkoholischer Lösung zu Trichlormethylsulfinsäure reducirt wird, wie folgende, von Loew) aufgestellte Gleichung zeigt.

$$
\begin{aligned}
\mathrm{CCl}_{3} \mathrm{SO}_{2} \mathrm{Cl}+\mathrm{H}_{2} \mathrm{~S} & =\mathrm{CCl}_{3} \mathrm{SOOH}+\mathrm{S}+\mathrm{HCl} . \\
\left(\mathrm{CCl}_{3} \mathrm{SO}_{2} \mathrm{Cl}+\mathrm{SO}_{2}+\mathrm{H}_{2} \mathrm{O}\right. & \left.=\mathrm{CCl}_{3} \mathrm{SOOH}+\mathrm{HCl}+\mathrm{SO}_{3}\right) .
\end{aligned}
$$

Ich möchte hier bemerken, dass diese Einwirkung, insofern sie Schwefelwasserstoff betrifft, durch die Art des Lösungsmittels beeinflusst wird, denn ich habe gefunden, dass auf eine Lösung des reinen Trichlormethylsulfonchlorids in kaltem absoluten Benzol reiner und trockner Schwefelwasserstoff gar keine reducirende Einwirkung ausübt. Um das ganz sicher festzustellen, liess ich die mit Schwefelwasserstoff übersättigte Lösung in einem fest verkorkten Probirröhrchen mehrere Tage stehen; nicht eine Spur Schwefel schied sich hierbei aus, und die Flüssigkeit roch noch immer stark nach Schwefelwasserstoff.

Einwirkung von Anilin auf Trichlormethylsulfonchlorid.

Die Wechselwirkung von Anilin und diesem Chlorid ist verschieden, je nachdem die beiden Körper bei Gegenwart oder Abwesenheit von Lösungsmitteln zusammengebracht werden;

1) Zeitschr. Chem. 1869, S. 82. 
insbesondere des Trichlormethylsulfonchlorids. 291

auch hängt dieselbe zum Theil von der Art des Lösungsmittels ab.

a. Ohne Lösungsmittel.

Die Reaction ist sehr heftig und geht manchmal bis zur Entwicklung von schwefliger Säure.

b. Lösung in Alkohol (oder Aether-Alkohol).

$\mathrm{Zu}$ einer Lösung von ungefähr $11 \mathrm{Grm}$. Chlorid in Aether wurden, nachdem alles Wasser durch Chlorcalcium entfernt und die Flüssigkeit filtrirt war, ungefähr $10 \mathrm{Ccm}$. Anilin (i. e. ein kleiner Ueberschuss) und etwas absoluter Alkohol hinzugefügt, und die zugestöpselte Flasche ein paar Stunden stehen gelassen. Der Aether wurde dain abdestillirt, und die alkoholische chocoladebraun gefärbte Lösung wieder über Nacht stehen gelassen. Auf Zusatz von Wasser - (ungefähr zwei Theile Wasser auf einen Theil Lösung) - schied sich eine grosse Menge einer rothbraunen, weichen, theerartigen Substanz aus (welche ich nicht weiter untersucht habe). Das Filtrat derselben wurde auf dem Wasserbade eingedunstet und dann ein paar Stunden in der Kälte stehen gelassen. Auf diese Weise wurde eine ziemliche Menge von Krystallen erhalten, welche ich durch Abpressen zwischen Papier, Wiederauflösen in Wasser, Kochen der wässrigen Lösung mit Thierkohle und schliesslich durch Umkrystallisiren aus einem Gemisch von zwei Theilen Benzol und einem Theil absoluten Alkohol reinigte. In Alkohol allein waren die Krystalle zu leicht löslich.

\section{Analyse:}

1) 0,1904 Grm. gaben, mit Kalk geglüht, 0,0728 Grm. Cl.

$$
\begin{array}{ccc} 
& \text { Berechnet } & \text { Grefunden. } \\
\text { Cl für } \mathrm{CCl}_{3} \mathrm{SO}_{2} \mathrm{NH}\left(\mathrm{C}_{8} \mathrm{H}_{5}\right) . & \\
\mathbf{3 8 , 8 0} \% & 38,24 \%
\end{array}
$$

2) $0,3595 \mathrm{Grm}$. gaben $16,85 \mathrm{Ccm}$. N bei $755 \mathrm{Mm}$. Bar. und $13^{\circ}$.

$$
\begin{array}{ccc}
\text { Berechnet } & \text { Gefunden. } \\
\mathrm{N} & 5,10 \% & \\
\mathrm{CCl}_{3} \mathrm{SO}_{2} \mathrm{NH}\left(\mathrm{C}_{6} \mathrm{H}_{5}\right) . & 5,50 \%
\end{array}
$$

3) 0,3263 Grm., mit chromsaurem Blei, Kupferoxyd und Kupfer verbrannt, gaben 0,3555 Grm. $\mathrm{CO}_{2}$ und 0,0940 Grm. $\mathrm{H}_{2} \mathrm{O}$. 
292 MeGowan: Ueb. d. Abkömml. d. Methylsulfonsäure,

$\begin{array}{ccc} & \text { Berechnet } & \text { Gefunden. } \\ & \text { für } \mathrm{CCl}_{3} \mathrm{SO}_{2} \mathrm{NH}\left(\mathrm{C}_{6} \mathrm{H}_{5}\right) . & \\ \text { C } & 29,71 \% & 30,60 \% \\ \text { H } & 2,15 \% & 3,20 \%\end{array}$

Diese Krystalle enthielten auch Schwefel, aber nicht als Schwefelsäure.

Das Trichlormethylsulfonanilid krystallisirt aus AlkoholBenzol in kleinen breiten Nadeln. In Alkohol sind die Krystalle sehr leicht, in Wasser ziemlich, in Benzol aber so gut wie gar nicht löslich.

\section{c. Lösung in Benzol.}

In diesem Falle wird gleichfalls das obige Anilid gebildet; zu gleicher Zeit macht sich stärkere Zorsetzung unter. Schwärzung bemerkbar. Alkohol ist deshalb als Lösungsmittel vorzuziehen.

\section{d. Lösung in Aether.}

Wird zu einer absolut ätherischen Lösung des Chlorids (ungefähr 1 Thl. Chlorid auf 6 Thle. Aether) ein kleiner Ueberschuss, nämlich etwas mehr als zwei Aequivalente von Anilin, hinzugefügt, die Flasche zugestöpselt und über Nacht stehen gelassen, so scheidet sich salzsaures Anilin aus (und zwar ein Aequivalent salzsaures Salz für jedes Aequivalent Chlorid), und die Lösung wird dunkelbraun. Wenn nun der Aether im Vacuum über Schwefelsäure verdunstet wird ${ }^{1}$ ), wobei mit dem Aether zugleich stechend riechende Dümpfe, welche die Augen sehr angreifen, entwickelt werden, so bleibt eine Masse, theils theerartig, theils krystallinisch, zurück. Dieses Gemisch wird am besten mit heissem Wasser, welches ein wenig Alkohol enthält, auf dem Wasserbade behandelt (wobei die übelriechenden Dämpfe wieder wahrnehmbar sind). Der grösste Theil der Theersubstanz ${ }^{2}$ ) bleibt

1) Nicht bei einer höheren 'I'emperatur; denn als ich bei einem Versuch die letzten Portionen Aether auf dem Wasserbare verjagte, trat eine sehr heftige Reaction ein. Unter starker Wärmeentwicklung entwichen stechende saure Dämpfe, während eine dunkel gefärbte theer- oder harzartige Masse zurückblieb.

2) Welche, der Luft ausgesetzt, hart und spröde wird. 
insbesondere des Trichlormethylsulfonchlorids. 293

mgelöst, and das Filtrat, nachdem es von den letzten Spuren derselben mittelst Thierkohle befreit worden ist, liefert durch Eindampfen zwei Arten Krystalle, nämlich strahlige undurchsichtige Warzen, und Nadeln von salzsaurem Anilin. Da letzteres viel leichter als jenes in Wasser löslich ist, so lassen sie sich ohne Mühe von einander trennen; der neue Körper wird leicht rein erhalten durch Umkrystallisiren aus $80 \%$ bis $90 \%$ Alkohol (in absolutem Alkohol ist er zu wenig löslich), aus welchem er beim Erkalten sich sofort in feinen glänzenden Nadeln abscheidet.

Analyse:

1) $0,2423 \mathrm{Grm}$. gaben $18,5 \mathrm{Ccm}$. N bei $753 \mathrm{Mm}$. Bar. und $10^{\circ}$. Dieses entspricht $9,06 \% \mathrm{~N}$.

2) Die wässrige Lösung des Salzes gab mit salpetersaurem Silber keine Chlorreaction.

0,1570 Grm. gaben, mit Kalk geglüht, 0,0694 Grm. AgCl, entsprechend 0,0171 Grm. $\mathrm{Cl}=10,93 \% \mathrm{Cl}$.

3) a. 0,1862 Grm., mit chromsaurem Blei, Kupferoxyd und Kupfer verbrannt, lieferten $0,3044 \mathrm{Grm} . \mathrm{CO}_{2}$ und $0,0826 \mathrm{Grm} . \mathrm{H}_{2} \mathrm{O}=44,59 \% \mathrm{C}$ und $4,93 \% \mathrm{H}$.

b. 0,1345 Grm. gaben 0,2238 Grm. $\mathrm{CO}_{3}$ und 0,0658 Grm. $\mathrm{H}_{2} \mathrm{O}=$ $45,38 \% \mathrm{C}$ und $5,44 \% \mathrm{H}$.

c. $0,1598 \mathrm{Grm}$. gaben $0,0721 \mathrm{Grm} . \mathrm{H}_{2} \mathrm{O}=5,01 \% \mathrm{H}$.

4) Die Verbindung enthielt auch Schwefel, und zwar nur als Schwefelsäure.

0,1316 Grm. in Wasser, welchem einige Tropfen verdünnter Salzsäure zugesetzt werden, aufgelöst und direct durch Chlorbarium gefällt, gaben 0,0952 Grm. Ba SO 4. Dieses entspricht 9, $94 \%$ (als $\mathrm{SO}_{3}$ ).

Diese Zahlen stimmen mit den für ein einfach-gechlortes schwefelsaures Anilin bereclmeten überein.

Berechnet

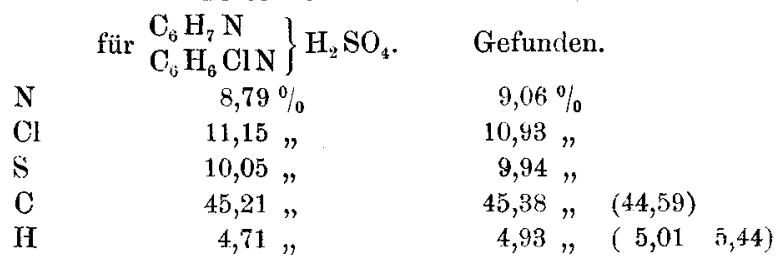

Diese Verbindung ist demnach als schwefelsaures Doppelsalz des Anilins und eines Chloranilins zu betrachten. 
294 Me G o wan: Ueb. d. Abkömml. d. Methylsulfonsäure,

Die complicirte Reaction, durch welche dasselbe entsteht, entzieht sich vorläufig der Erklärung. Das Trichlormethylsulfonchlorid hat ohne Zweifel auf einen Theil des Anilins chlorirend eingewirkt.

Einwirkung von Ammoniak auf Trichlormethylsulfonchlorid.

a. In wässriger Lösung.

Die aussergewöhnliche, in ihrer Art einzige Einwirkung wässrigen Ammoniaks auf dieses Chlorid wurde von Loew ${ }^{1}$ ) beobachtet. Er fand, dass das letztere zu Sulfinsäure, unter Entwicklung von Stickstoff, reducirt wird, nach folgender Gleichung:

$$
3 \mathrm{CCl}_{3} \mathrm{SO}_{2} \mathrm{Cl}+8 \mathrm{NH}_{3}=\underbrace{3 \mathrm{CCl}_{3} \mathrm{SOONH}_{4}}_{\begin{array}{c}
\text { Trichlormethylsulfin- } \\
\text { saures Ammon. }
\end{array}}+\mathrm{N}_{3}+3 \mathrm{NH}_{4} \mathrm{Cl} .
$$

Ich habe die Einwirkung von Ammoniak unter verschiedenen Bedingungen eingehend untersucht, und zwar immer mit demselben Resultat. Es ist mir nicht gelungen, das A mid der Trichlormethylsulfonsäure darzustellen.

Einige quantitative Bestimmungen des durch die Einwirkung von wässrigem Ammoniak auf das Chlorid entwickelten Stickstoffs stimmten mit den nach Loew's Gleichung berechneten Mengen annähernd überein. ${ }^{2}$ ) und $10^{\circ}$.

1) 1,871 Grm. Chlorid gaben $62,5 \mathrm{Ccm}$. $\mathrm{N}$ bei $759 \mathrm{Mm}$. Bar.

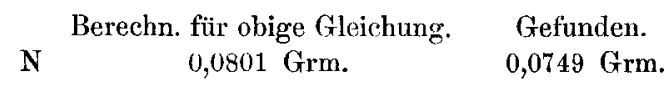

1) Zeitschr. Chem. 1869, S. 82.

2) Das Ende des Rohrs, durch welches der Stickstoff aus der Entwicklungstlasche herausgeht, muss fast zu einer Capillare ausgezogen werden, um das mechanische Uebergehen von kleinen Partikeln des Chlorids zu vermeiden. Gegen Ende der Reaction wird die Flasche erwärmt.

Es ist nicht unwahrscheinlich, dass ausser obiger Einwirkung (ine kleine Quantität des Chlorids mit dem Wasser Trichlormethylsulfonsiure erzengt, und dass deshalb nicht die ganze berechnete Menge Stickstoff gewonnen wird. 
insbesondere des 'Trichlornethylsulfonchlorids. 295 und $12^{\circ}$.

2) 1,1973 Grm. Chlorid gaben $37,8 \mathrm{Ccm}$. $\mathrm{N}$ bei $748 \mathrm{Min}$. Bar.

$$
\text { N } \quad 0,0513 \text { Grm. } \quad 0,0442 \text { Grm. }
$$

b. In alkoholischer Lösung.

Ganz dieselbe Einwirkung, wie bei Anwendung von wässrigem Ammoniak findet hier statt; die Reaction ist auch in diesem Falle ziemlich energisch.

\section{c. In Benzollösung.}

Die Einwirkung schreitet hier viel langsamer vorwärts als in den zwei obigen Fällen, ist jedoch schliesslich dieselbe. Ich untersuchte sie unter verschiedenen Bedingungen.

1) Auf in einem graduirten Rohre über Quecksilber aufbewahrtes trocknes Ammoniakgas liess ich eimen Ueberschuss einer Benzollösung des reinen Chlorids einwirken. (Z. B. auf ungefähr $100 \mathrm{Ccm}$. Ammoniak brauchte ich etwa 1,3 Grm. Chlorid in 4 bis $5 \mathrm{Ccm}$. Benzol aufgelöst.) Die Absorption erfolgte langsam und bedurfte länger, als einen halben Tag zur Vollendung. Endlich blieb ein Rest von Stickstoff zurück. Um festzustellen, dass dieses kein Ammoniak mehr enthielt, brachte ich jetzt in das Rohr etwas mit Alkohol versetzte verdünnte Schwefelsäure und schüttelte gut um. Die Quantität Stickstoff, welche erhalten wurde, war auch hier etwas geringer als die berechnete; bei einem Versuche fand ich 7,79 Ccm. statt 9,8 Ccm. N.

2) Eine kleine Quantität (etwa $1-2$ Grm.) des reinen Chlorids wurde der Finwirkung von einem grossen Ueberschuss einer ammoniakalischen Benzollösung ausgesetzt, und das daraus entwickelte Gas über Benzol in einem graduirten Rohre angesammelt. Die Einwirkung verlief in diesem Fallo langsamer wie oben und brauchte mehrere Tage.

d. Ohne Lösungsmittel.

Ein Uebersehuss des reinen Chlorids, auf Ammoniakgas üher Quecksilber einwirkend, macht ebenfalls Stickstoff frei. Dicse Absorption schreitet viel schneller fort, als jene mit ein^r Benzollösung des Chlorids.

Demnach haben die für das Ammonials gebrauchten 
296 M:Gowan: Ueb. d. Abkömml. d. Methylsulfonsäure,

Lösungsmittel wohl auf die Schnelligkeit jener eigenthümlichen Reaction Eirffluss, jedoch wird dadurch dieselbe in ihrer Art nicht verändert. Das Trichlormethylsulfonamid, dessen Entstehung man erwarten sollte, scheint nach Allem nicht existenzfähig zu sein. $\left.{ }^{1}\right\rangle$

Einwirkung von Methylamin auf Trichlormethylsulfonchlorid.

Lässt man einen Ueberschuss der concentrirten wässerigen Lösung von Methylamin auf das Chlorid in der Kälte einwirken, so löst sich letzteres allmählich auf, und ebenso wie bei der Einwirkung von Ammoniak wird Stickstoff frei. Ich machte einige approximative Bestimmungen der Menge dabei entwickelten Stickstoffs.

1) 0,6365 Grm. reines Chlorid mit etwa 1,2 Cem. 30 proc. Methylamin gaben $10,3 \mathrm{Ccm}$. $\mathrm{N}$ bei $754 \mathrm{Mm}$. Bar. und $25^{\circ}=1,79 \% \mathrm{~N}$ (des Gewichts des gebrauchten Chlorids).

2) 0,6433 Grm. Chlorid mit 1,3 Ccm. Methylamin gaben $9,7 \mathrm{Ccm}$. $\mathrm{N}$ bei $751 \mathrm{Mm}$. Bar. und $22^{\circ}=1,69 \% \mathrm{~N}$.

Die letzten Portionen Gas wurden durch Erwärmen aus der kleinen Flasche ansgetrieben. Ich bemerkte auch hier, dass einige Tropfen éner ätherischen Flüssigkeit überdestillirten. Die Produkte dieser Reaction sollen noch genauler untersucht werden.

\section{Derivate der Dichlormethylsulfonsäure: $\mathrm{CCl}_{2} \mathrm{HSO}_{2} \mathrm{OH}$.}

Wir lernen aus obigen Versuchen, dass ein bedeutungsvoller Unterschied zwischen dem Verhalten des Methylsulfonchlorids und dem des Trichlormethylsulfonchlorids besteht. Ersteres verhält sich wie ein normales Säurechlorid; es lässt sich durch Einwirkung von Fïnffach-Chlorphosphor auf

1) Es wäre viellcicht nicht ohne Interesse, einige weitere Versuche über die Einwirkung des Ammoniaks auf das 'Trichlormethylsulfonchlorid bei sehr niedriger 'Temperatur anzustellen.

Es mag hier erwähnt werden, dass das trichlormethylsulfinsaure Ammon, welches aus Wasser in schönen Blattern sich ausscheidet, aus Benzol in langen dünnen Prismen krystallisirt. 
insbesondere des Trichlormethylsulfonchlorids. 297

die Methylsulfonsäure darstellen, wird nicht von Schwefelwasserstoff reducirt, und liefert mit Ammoniak das zugehörige Amid. Das Trichlormethylsulfonchlorid dagegen kann nicht aus der Säure, resp. ihren Salzen, durch Einwirkung von Fünffach-Chlorphosphor bereitet werden, es wird in alkoholischer Lösung von Schwefelwasserstoff leicht zu der entsprechenden Sulfinsäure reducirt, und giebt mit Ammoniak, unter Stickstoffentwicklung, gleichfalls Sulfinsäure.

Naturgemäss drängt sich hier die Frage auf, wie werden sich die zwischen jenen Chloriden liegenden Mono- und Dichlormethylsulfonchloride unter gleichen Bedingungen verhalten, oder, mit anderen Worten, werden beide letzteren dem Trichlormethylsulfonchlorid oder einem normalen Säurechlorid analog sein? Um diese Fragen zu lösen, stellte ich folgende Versuche an.

Dichlormethylsulfonsäure: $\mathrm{CCl}_{2} \mathrm{H} \mathrm{SO}_{2} \mathrm{OH}$.

Kolbe ${ }^{1}$ ) hat beobachtet, dass durch Einwirkung von Zink auf Trichlormethylsulfonsäure die letztere zu Dichlormethylsulfonsäure (resp. deren Zinksalz) ohne Wasserstoffentwicklung reducirt wird, und ferner, dass die nämliche Säure auch leicht erhalten werden kann durch Behandeln des Produkts der Reduction von Trichlormethylsulfonchlorid durch Schwefelwasserstoff (i. e. Trichlormethylsulfinsäure) mit Kalilauge, gemäss der Gleichung:

$$
\mathrm{CCl}_{3} \mathrm{SOOH}+2 \mathrm{KOH}=\mathrm{KCl}+\mathrm{H}_{2} \mathrm{O}+\mathrm{CCl}_{2} \mathrm{HSO}_{2} \mathrm{OK} \text {. }
$$

Ich fand letztere Methode ganz unbefriedigend. Nachdem die Lösung des Trichlormethylsulfonchlorids in absolutem Alkohol durch schweflige Säure reducirt, und der Ueberschuss letzterer durch einen Strom Kohlensäure ausgetrieben war, fügte ich zu der alkoholischen Lösung etwas Wasser und dann Aetzkali in kleinen Quantitäten hinzu, so dass die Flüssigkeit immer alkalisch blieb, und digerirte auf dem Wasserbade, bis keine Trichlormethylsulfinsäure mehr vorhanden war. Dies liess sich leicht durch Zusatz von Bromwasser zu einer Probe der Lösung erkennen; so lange

1) Ann. Chem. Pharm. 54, 145. 
298 MeGowan: Ueb. d. Abkïmml. d. Methylsulfonsäure, jene Sulfinsäure dabei war, bekam man einen wcissen Niederschlag von Trichlormethylsulfonbromid, $\mathrm{CCl}_{3} \mathrm{SO}_{2} \mathrm{Br}$. Sobald letzterer nicht mehr entstand, neutralisirte ich den Jeberschuss vorhandenen Kalis mit Kohlensäure, dampfte auf dem Wasserbade ein und zog den Rückstand mit 90 proc. Alkohol aus. Um die Zusammensetzung des resultirenden Salzes festzustellen, wurde es zweimal aus 90 proc. Alkohol umkrystallisirt und dann analysirt. $\mathrm{K}, \mathrm{SO}_{4}$.

1) 0,0991 Grm. gaben, mit Schwefelsüure erhitzt, 0,0592 Grm.

Berechnet für

Gefunden.

$\begin{array}{cccc} & \mathrm{C} \mathrm{Cl}_{2} \mathrm{HSO}_{2} \mathrm{OK} & \mathrm{CClH}_{2} \mathrm{SO}_{2} \mathrm{OH} & \mathrm{CH}_{3} \mathrm{SO}_{2} \mathrm{OK} \\ \mathrm{K} & 23,1 \% & 29,1 \% & \\ 19,21 \% & & 26,8 \%\end{array}$

2) Nach dieser Kalibestimmung krystallisirte ich eine andere kleine Quantität des Salzes aus kochendem absoluten Alkohol um, für eine

Chlorbestimmung:

0,1917 Grm., mit Kalk geglüht, gaben 0,01074 Grm. Cl.

Berechnet für

Gefunden.

$\mathrm{CCl}_{2} \mathrm{HSO}_{2} \mathrm{OK} \quad \mathrm{CClH}_{2} \mathrm{SO}_{2} \mathrm{OK} \quad \mathrm{CH}_{3} \mathrm{SO}_{2} \mathrm{OH}$

Cl $\quad 34,98 \%$

$20,46 \%$

$5,60 \%$

Daraus folgt, dass dieses Produkt hauptsächlich aus methylsulfonsaurem Kali besteht, welchem eine kleire Quantität von einem oder von beiden der oben genannten chlorirten Salze beigemengt ist, dass also die Entziehung von Chlor aus trichlormethylsulfinsaurem Kali zu weit gegangen ist. $\left.{ }^{1}\right)$

Die Methode zur Bereitung der Dichlormethylsulfonsäure, durch Reduction der wässrigen Lösung der Trichlormethylsulfonsäure mittelst Zink, ist der zuletzt besprochenen weit vorzuziehen, die Einwirkung ist fast eine quantitative. ${ }^{2}$ ) Gegen Ende der Reaction wird diese zweckmässigerweise

1) Ich habe es noch nicht versucht, das Trichlormethylsulfinsalz nur in wässuiger Lösung durch Kali zu entchloren; die Einwirkung würde hier natürlich eine mässigere sein.

2) Ich fand allemal, dass hier viel Wasserstoff entwickelt wird. 
insbesonclere des Trichlormethylsulfonchlorids. 299

durch Erwärmen auf dem Wasserbade befördert. Folgende Versuche dienen als Belege.

1) $20 \mathrm{Grm}$. Trichlormethylsulfonsäure lösten $5,8 \mathrm{Grm}$. Zink auf; die nach der Gleichung berechnete Menge ist 6,5 Grm.

2) $60,2 \mathrm{Grm}$. Säure lösten 18,3 Grm. Zink auf; berechn, 19,6 Grm.

Aus der resultirenden Lösung des Zinksalzes bereitete ich die Säure genau nach Kolbe's ${ }^{1}$ ) Vorschrift; das Zink wurde durch kohlensaures Kali ausgefällt, das Filtrat von kohlensaurem Zink bis zur Trockne auf dem Wasserbade verdunstet, und der Rückstand mit Alkohol von ungefähr $80 \%$ ausgezogen.

Ich reinigte eine Portion dieses Kalisalzes durch Umkrystallisiren aus Alkohol und machte folgende Kalibestimmung davon:

0,2280 Grm. gaben 0,0990 Grm. $\mathrm{K}_{2} \mathrm{SO}_{4}$.

$$
\begin{array}{cc}
\text { Berechn. für } \mathrm{CCl}_{2} \mathrm{HSO}_{2} \mathrm{OK} . & \text { Gefunden. } \\
19,47 \% & 19,41 \%
\end{array}
$$

Aus dem alkoholischen Extract wurde das Kali durch verdünnte Schwefelsäure als schwefelsaures Salz abgeschieden $\left.{ }^{2}\right)$, dieses abfiltrirt, und in dem Filtrat, nach Zusatz von Wasser und Abdestilliren des darin enthaltenen Alkohols, der Ueberschuss von Schwefelsäure so genan wie möglich durch Barythydrat ansgefällt. Das Filtrat von dem schwefelsauren Baryt lieferte nach Eindampfen auf dem Wasserbade die Dichlormethylsulfonsäure ziemlich rein.

\section{Dichlormethylsulfonchlorid: $\mathrm{CCl}_{2} \mathrm{HSO}_{2} \mathrm{Cl}$.}

Fünffach-Chlorphosphor wirkt auf Dichlormethylsulfonsäure bei gewöhnlicher Temperatur leicht ein; die Reaction wurde auf dem Wasserbade beendet. Nachdem der grösste Theil des Phosphoroxychlorids abdestillirt war, wurde zu der

1) Ann. Chem. Pharm. 34, 145.

2) Wird zu einer alkoholischen Lösung eines organischen Kalisalzes in der Kälte oder Wärme verdünnte Schwefelsäure hinzugesetzt, so fällt neutrales schwefelsaures Kali nieder, selbst wenn Schwefelsäure genug zugegen ist, um das saure Salz zu bilden. Dieses wurde bestätigt: 1) mit einer heissen Lösung von dichlormethylsulfonsaurem Kali in $80 \%-90 \%$ Alkohol; 2) mit einer kalten Lösung von essigsaurem Kali in $80 \%-90 \%$ Alkohol. 
300 M:Gowan: Ueb. d. Ablömml. d. Mothylsulfonsäure, zurückbleibenden Flüssigkeit eine genügende Menge Eis linzugefügt, um div letzten Theile des Phosphoroxychlorids z.u zerstören, und dann der Rückstand mittelst Chlorealcium getrocknet. (Ich hatte zu wenig Substanz, um sie durch Fractioniren reinigen zu können.)

Analyse:

0,4669 Grm, obiger Flüssigkeit, mit Kalk geglüht, gaben 0,2741 (y)m. Cl.

$$
\begin{aligned}
& \text { Berechn. fǜr } \mathrm{C} \mathrm{Cl}_{2} \mathrm{HSO}_{2} \mathrm{Cl} \text {. Gefunden. } \\
& \text { Cl } \quad 58,04 \% \quad 58,70 \%
\end{aligned}
$$

Das auf obige Weise gewomnene Liquidum ist demnach Dichlormethylsulfonchlorid; aus seiner Darstellung erhellt, lass die Dichlormethylsulfonsäure in normaler Weise von Fünffach-Chlorphosphor angegriffen wird. (Unterschied von der Trichlormethylsulfonsäure.)

Dichlormethylsulfonchlorid ist eine Flüssigkeit von scharfem. Geruch, aber nicht so stechend, wie der vom Methylsulfonchlorid. An feuchter Luft bildet es Dämpfe. Sein spec. Gewicht beträgt 1,71. Es scheint zwischen $170^{\circ}-180^{\circ}$ zu sieden (zu einer genauen Bestimmung des Kochpunkts fehlte mir Material). Der Versuch, dieses Dichlormethylsulfonchlorid durch Erhitzen mit Dreifach-Chlorjod in einem geschlossenen Rohre wieder in das Trichlormethylsulfon- . chlorid zurückzuverwandeln, war erfolglos. Bis zu einer Temperatur von $130^{\circ}$ schien keine Einwirkung stattzufinden; nach weiterem Erhitzen auf $230^{\circ}$ hatte das Chlorid sich in schweflige Säure und (wabrscheinlich) einen Chlorkohlenstoff zcrsetzt. Bei so hoher Temperatur konnte das etwa gebildete Trichlormethylsulfonchlorid nicht bestehen.

\section{Einwirkung von Ammoniak auf Dichlormethyl- sulfonchlorid.}

Trockenes Ammoniakgas, in einem graduirten Rohre über Quecksilber aufgesammelt, wurde von einer Benzollösung des Chlorids vollständig absorbirt; Stickstoff bildete sich also nicht. 
Dichlormethylsulfonamid: $\mathrm{CCl}_{2} \mathrm{HSO}_{2} \mathrm{NH}_{2}$.

Wird trocknes Ammoniak durch eine Lösung des Dichlormethylsulfonchlorids in Benzol geleitet, so entsteht eine halbfeste Masse unter Freiwerden von Wärme. Um die Einwirkung vollständig zu machen, fügte ich schliesslich zu diesem Gemisch etwas Alkohol und leitete dazu Ainmoniakgas bis zur Sättigung.

Nach Ausziehen des Ganzen mit einem heissen Gemisch von absolutem Alkohol und Benzol und nach Eindampfen des Auszugs, schliesslich im Vacuum über Schwefelsäurc, blieb eine farblose syrupartige Masse zurück. Diese wurde von Spuren von Chlorammonium durch Wiederauflösen in einem kalten Gemisch von zwei Theilen Benzol und einem Theil absoluten Alkohol befreit.

Analyse des Rückstandes dieser Lösung:

1) 0,4724 Grm. gaben $35,7 \mathrm{Ccm}$. $\mathrm{N}$ bei $752 \mathrm{Mm}$. Bar. und $18^{\circ}$.

$$
\mathrm{N} \begin{array}{cc}
\text { Berechn. für } \mathrm{CCl}_{2} \mathrm{HSO}_{2} \mathrm{NH}_{2} \text {. Gefunden. } \\
8,54 \% & 8,64 \%
\end{array}
$$

2) 0,4174 Grm., mit Kalk geglüht, gaben 0,1833 Grm. Ul.

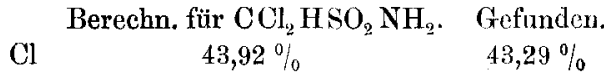

Dieses ist demnach Dichlormethylsulfonamid. Nach längerem Stehen über Schwefelsäure krystallisirte es in sehr zerfliesslichen langen dünnen Prismen.

Einwirkung von schwefliger Säure auf Dichlormethylsulfonchlorid in absolutem Alkohol.

Zu etwa $13 \mathrm{Ccm}$. abgekühlten absoluteı Alkohols wurde tropfenweise $1 \mathrm{Ccm}$. von dem Dichlormethylsulfonchlorid gesetzt und dazu - immer unter guter Abkühlung - schweflige Säure bis zur Sättigung geleitet.

Durch sorgfältiges Abkühlen sollte eine mögliche Wechselwirkung zwischen dem Chlorid und dem Alkohol vermiede. werden. Dann wurde das zugestöpselte Probirrohr über Nacht stehen gelassen. Am folgenden Tage erwärmte ich die Lösung auf dem Wasserbade, um den Ueberschuss von schwefliger Bäure zu verjagen, neutralisirte mit kohlensaurem 
302 M:G owan: Ueb. d. Abkömml. d. Methylsulfonsäure,

Kali unter Zusatz von ein wenig Wasser und extrahirte den Rückstand mit kochendem Alkohol von etwa $95 \%$. Nach öfterem Umkrystallisiren bekam ich ein Salz, welches gleichmässig in langen, vielfach gekreuzten Prismen krystallisirte. Dieses über Schwefelsäure und dann bei $100^{\circ}$ getrocknete Salz wurde durch Erhitzen mit Salpetersäure von 1,4 spec. Gew. auf $100^{\circ}$ nicht verändert.

Analyse:

0,0974 Grm. desselben gaben 0,0456 Grm. $\mathrm{K}_{2}, \mathrm{SO}_{4}$.

$$
\text { K Berechn. } 20,86 \% \text {.ü } 20,99 \%
$$

Das so gewonnene Salz krystallisirte ganz anders als das Salz, welehes durch Einwirkung von wässrigem kohlensauren Kali anf Dichlormethylsulfonchlorid und nachheriges Eindampfen und Ausziehen mit absolutem Alkohol dargestellt ist. Diese letzteren Krystalle von dichlormethylsulfonsaurem $\mathrm{Kali}, \mathrm{COl}_{2} \mathrm{HSO}_{2} \mathrm{OK}$, sind sehr dünne, glänzende Blätter, wie sie von $\mathrm{K}_{0 l b e^{1}}$ ) beschrieben worden sind.

Einwirkung von Schwefelwasserstoff anf Dichlormethylsulfonchlorid.

a. In Benzollösung.

Schwefelwasserstoff hat auf eine Lösung des Dichlormetlylsulfonchlorids in Benzol gar keine reducirende Einwirkung; selbst nach längerem Stehen der mit dem Gas gesüttigten Lösung scheidet sich kein Schwefel ab.

\section{b. Tn alkoholischer Lösung}

aber tritt eine sehr langsame Reaction unter Ausscheidung von Schwefel ein. Der Schwefelwasserstoff muss mehrere Tage durch die Lösung geleitet werden, bis sich kein Schwefel mehr ausscheidet, und die Lösung noch immer stark nach dem Gase riecht. Ausser dem Schwefel setzten sich einige lange farblose, gekreuzte Prismen ( $\mathrm{CCl}_{2} \mathrm{HSOOH}$ ?) $a b$, welche aber leicht durch gelindes Erwärmen wieder gelöst wurden. Nachdem der Ueberschuss von Schwefelwasser-

1) Ann. Chem. Pharm. 54, 145. 
insbesondere des Trichlormethylsulfonchlorids. 303 stoff durch Kohlensäure vertrieben war, wurde die sodann mit kohlensaurem Kali neutralisirte Lösung eingedampft, und der Rückstand aus Alkohol umkrystallisirt (wie oben angegeben).

Das so erhaltene Salz hat genau dieselbe Form, wie las durch die Einwirkung von schwefliger Säure gewonnene; es wurde ebenfalls nicht durch Salpetersäure von 1,4 spec. Gew. verändert.

Ich musste, wegen Mangels an Substanz mit allzu kleinen Quantitäten des Chlorids arbeiten; zudem war die Ansbeute an dem Salze so gering, dass ich mich nicht ausführlicher über die obigen Reactionen äussern mag, bis ich den Versuch wiederholt habe. Die Thatsache, dass concentrirte Salpetersäure keine Einwirkung übt, spricht zunächst gegen die Annahme, dass dasselbe ein sulfinsaures Salz ist. Ich hoffe, bald diesen Punkt aufklären zu können.

Die wichtigsten, aus den vorhergehenden Versuchen sicls ergebenden Folgerungen können in nachstehenden Sätzen zusammengefasst werden.

1) Die Methylsulfonsäure und die Dichlormethylsulfonsäure geben mit Fünffach-Chlorphosphor die entsprechenden Süurechloride; Trichlormethylsulfousäur: dagegen lässt sich nicht in ihr Chlorid umwandeln.

2) Auf keine Weise ist es bis jetzt gelungen, die $\mathrm{Mc}$ thylsulfonsäure oder deren Chlorid in Chlorderivate überzuführen.

3) Cyankalium scheint nicht direkt auf Methylsulfonchlorid einzowirken; mit Trichlormethylsulfonchlorid giebt es Trichlormethylsulfinsäure unter Entwickelung von Chlorcyan.

4) Schwefelwasserstoff hat auf Methylsulfonchlorid in alkoholischer Lösung keine reducirende Wirkung; dagegen reducirt derselbe (sowie schweflige Säure) das Trichlormethylsulfonchlorid zu der entsprechenden Sulfinsäure. Auch das Dichlormethylsulfonchlorid erfährt höchst wahrscheinlich durch Schwefelwasserstoff Reduction. Die Natur des Lösungsmittels ist hier von Einfluss, da diese Reduction bei An- 
304 McGowa n: Ueb.d. Abkömml.d. Methylsulfonsäure etc.

wendung von Benzol statt Alkohol in keinem der beiden obigen Fällen stattfindet.

5) Ammoniak liefert mit dem Methylsulfonchlorid und dem Dichlormethylsulfonchlorid die entsprechenden Amide, mit Trichlormethylsulfonchlorid dagegen giebt es unter allen Umständen kein Amid, sondern ein Reductionsprodukt, nämlich Trichlormethylsulfinsäure, unter Stickstoffentwickelung. Diese, zuerst yon Loew beobachtete Reaction ist bislang einzig in ihrer Art.

Die Schnelligkeit der Reaction hängt davon $a b$, ob ein Lösungsmittel gebraucht wird oder nicht und ferner in ersterem Falle von der Natur des Lösungsmittels. Der chemische Vorgang ist aber schliessich derselbe.

6) Anilin giebt sowohl mit Methylsulfonchlorid als auch mit Trichlormethylsulfonchlorid die zugehörigen Anilide. Der Verlauf der Einwirkung auf das Trichlormethylsulfonchlorid ist jedoch von der Natur des angewandten Lösungsmittels abhängig. Ist letzteres Aether, so resultirt schwefelsaures Anilin-Chloranilin.

7) Metalle wirken auf Trichlormethylsulfonsäure verschieden ein; Eisen z. B. bildet damit trichlormethylsulfonsaures Salz, während Zink unter Reduction Dichlurmethylsulfonsäure liefert.

8) Aus Allem ergiebt sich, dass zwischen dem Trichlormethylsulfonchlorid und dem Methylsulfonchlorid, sowie den intermediären Chloriden, ein bedeutender Unterschied bestelt. -

Der Eintritt des dritten At. Chlor und damit die Bildung einer Trichlormethylverbindung hat die Natur des Produkts in mannigfacher Hinsicht geändert.

Ich beabsichtige, diese Arbeit fortzusetzen.

Leipzig, Kolbe's Laboratorium, August 1884. 